\title{
ON PSEUDOCONVEX FUNCTIONS AND APPLICATIONS TO GLOBAL OPTIMIZATION
}

\author{
A. HASSOUNI ${ }^{1}$ AND A. JADDAR ${ }^{1}$
}

\begin{abstract}
In this paper, we characterize pseudoconvex functions using an abstract subdifferential. As applications, we also characterize maxima of pseudoconvex functions, and we study some fractional and quadratic optimization problems.
\end{abstract}

Résumé. Nous caractérisons des fonctions pseudoconvexes en utilisant un sous différentiel abstrait. Comme applications, nous caractérisons également des maximums des fonctions pseudoconvexes, et nous étudions quelques problèmes d'optimisation fractionnaires et quadratiques .

\section{INTRODUCTION}

The pseudoconvexity notion that has been introduced first by Mangasarian in [17] has many applications in programming and mathematical economy. We will generalize some results of $[4,18,19]$, where the authors have characterized a pseudoconvex function supposed to be radially continuous or radially non-constant. After recalling some preliminary results in section 2 , we give in section 3 some results extending those of $[1,2,4,8,19]$ for classes of functions that are less regular and where the assumptions of radial continuity and radial nonconstancy are not always used. In section 4, we characterize maxima of pseudoconvex functions on convex sets. In section 5, we illustrate the theoretical results with two particular examples: a fractional and a quadratic problems.

\section{Some PRELIMINARY NOTIONS AND RESUlts}

In the sequel, by $X$ we mean a Banach space and $X^{*}$ its dual for the duality pairing $\langle.,$.$\rangle . For x \in X$ and $\varepsilon>0$, we denote by $B_{\varepsilon}(x)$ the "open" ball of center $x$ and radius $\varepsilon$. And for $x, y \in X$, the closed interval $[x, y]$ is the set

$$
\{t x+(1-t) y: \quad 0 \leq t \leq 1\} .
$$

For $x \neq y$ the semi-closed intervals $(x, y],[x, y)$ and the open interval $(x, y)$ are defined similarly by dropping one or two end-points. For any $A \subset X$, we denote by $\operatorname{int}(A)$ its interior and by $c l(A)$ its closure.

Let us recall that for any nonempty subset $C$ of $X$ and any point $x$ of $X$, the normal cone to $C$ at $x$ is defined by

$$
N(C, x)=\left\{x^{*} \in X^{*}: \forall y \in C,\left\langle x^{*}, y-x\right\rangle \leq 0\right\}
$$

\footnotetext{
${ }^{1}$ Département de mathématiques et d'Informatique, Faculté des Sciences, B.P. 1014, Rue Ibn Battouta,Rabat, Maroc; e-mail: hassouni@fsr.ac.ma \& jaddar@fsr.ac.ma

(C) EDP Sciences, SMAI 2007
} 
Consider a function $f: X \rightarrow \mathbb{R} \cup\{+\infty\}$, with a nonempty domain

$$
\operatorname{dom} f=\{x \in X: \quad f(x)<+\infty\} .
$$

For $\lambda \in \mathbb{R}$, the sublevel set $S_{f}(\lambda)$ is defined by

$$
S_{f}(\lambda)=\{x \in X: \quad f(x) \leq \lambda\} .
$$

The function $f$ is said to be quasiconvex if for any $x, y \in X$ we have:

$$
\text { for any } z \in[x, y], \quad f(z) \leq \max \{f(x), f(y)\} \text {. }
$$

And it is strictly quasiconvex if the above inequality is strict when $x \neq y$ and $z \in(x, y)$. The abstract subdifferential we consider here is defined as follows:

Definition 2.1. An operator $\partial$ that associates with any l.s.c. function

$f: X \rightarrow \mathbb{R} \cup\{+\infty\}$ and a point $x \in X$ a subset $\partial f(x)$ of $X^{*}$ is a subdifferential if the following assertions hold:

(P1) $\partial f(x)=\left\{x^{*} \in X^{*}: \quad f(y) \geq f(x)+\left\langle x^{*}, y-x\right\rangle \quad \forall y \in X\right\}$

when $f$ is convex.

(P2) If $x \in \operatorname{dom} f$ is a local minimum of $f$, then $0 \in \partial f(x)$.

(P3) $\partial f(x)=\partial g(x)$, for any $g: X \rightarrow \mathbb{R} \cup\{+\infty\}$ such that

$(f-g)$ is constant in a neighborhood of $x$.

(P4) $\partial f(x)=\emptyset$, for any $x \in X$ such that $f(x)=+\infty$.

In general, people working on the Mean Value Theorem know that to each kind $\partial$ of subdifferential corresponds a particular type of Banach space called $\partial$-reliable, in which this Theorem is valid.

Definition 2.2. [18] A Banach space $X$ is called $\partial$-reliable if for each l.s.c. function $f: X \rightarrow \mathbb{R} \cup\{+\infty\}$, for any Lipschitz convex function $g$ and any $x \in \operatorname{dom} f$ such that $f+g$ achieves its minimum in $X$ at $x$ and each $\varepsilon>0$, we have:

$$
0 \in \partial f(u)+\partial g(v)+B_{\varepsilon}^{*}(0),
$$

where $u, v \in B_{\varepsilon}(x)$ such that $|f(u)-f(x)|<\varepsilon$ and $B_{\varepsilon}^{*}(0)$ is the "open" ball of $X^{*}$ with center 0 and radius $\varepsilon$.

Indeed we have the fundamental result.

Theorem 2.1. [19] Let $X$ be a d-reliable space and let $f: X \rightarrow \mathbb{R} \cup\{+\infty\}$ be a l.s.c. function. For any $a, b \in \operatorname{domf}$ with $a \neq b$, there exist a sequence $\left(c_{n}\right)$ in $X$ converging to some $c \in[a, b)$ and a sequence $c_{n}^{*}$ in $\partial f\left(c_{n}\right)$ such that

$$
\begin{aligned}
& \text { i) } \quad \liminf _{n}\left\langle c_{n}^{*}, b-a\right\rangle \geq f(b)-f(a) . \\
& \text { ii) } \quad \liminf _{n}\left\langle c_{n}^{*}, \frac{\|b-a\|}{\|b-c\|}\left(b-c_{n}\right)\right\rangle \geq f(b)-f(a) .
\end{aligned}
$$

In the sequel we will use the "dag subdifferential"

$$
\partial^{\dagger} f(x)=\left\{x^{*} \in X^{*}: \quad\left\langle x^{*}, v\right\rangle \leq f^{\dagger}(x, v) \quad \forall v \in X\right\},
$$

where

$$
f^{\dagger}(x, v)=\limsup _{(t, y) \rightarrow\left(0_{+}, x\right)} t^{-1}(f(y+t(v+x-y)-f(y)) .
$$


It is a subdifferential introduced by Penot (see [18]) that is large enough to contain the Clarke-Rockafellar $\partial^{C R}$ and the Upper Dini $\partial^{D_{+}}$subdifferentials and still has good properties.

Recall that an operator $T: X \rightarrow 2^{X^{*}}$ is quasimonotone if for any $x, y \in X$ the following implication holds

$$
\left(\exists x^{*} \in T(x):\left\langle x^{*}, y-x\right\rangle>0\right) \Longrightarrow\left(\forall y^{*} \in T(y):\left\langle y^{*}, y-x\right\rangle \geq 0\right) .
$$

We have then the following relation between quasimonotonicity and quasiconvexity :

Theorem 2.2. $[\mathbf{1 8}, \mathbf{1 9}]$ Let $X$ be a Banach space and let $f: X \rightarrow \mathbb{R} \cup\{+\infty\}$ be a l.s.c. function. Consider the following assertions

i) $f$ is quasiconvex.

ii) $\partial f$ is quasimonotone.

Then i) implies ii) if $\partial f \subset \partial^{\dagger} f$.

And ii) implies i) if $X$ is $\partial$-reliable.

\section{Characterizations of pseudoconvex functions}

In this section we study some properties of pseudoconvex functions.

Recall that a function $f: X \rightarrow \mathbb{R} \cup\{+\infty\}$ is pseudoconvex for the subdifferential $\partial$ if for any $x, y \in X$ the following implication holds

$$
\left(\exists x^{*} \in \partial f(x):\left\langle x^{*}, y-x\right\rangle \geq 0\right) \Longrightarrow f(x) \leq f(y) .
$$

The function $f$ is strictly pseudoconvex if the right inequality that appears in the above implication is strict when $x \neq y$.

If $C$ is an open convex set of $X$, then we say that $f: C \rightarrow \mathbb{R} \cup\{+\infty\}$ is pseudoconvex (respectively strictly pseudoconvex) if the function defined by

$$
\hat{f}(x)= \begin{cases}f(x) & \text { in } C, \\ +\infty & \text { otherwise } .\end{cases}
$$

is pseudoconvex (respectively strictly pseudoconvex) on $X$.

We can easily check that a pseudoconvex function $f$ on $X$ is pseudoconvex on any open convex subset $C$ of $X$. There is a close link between pseudoconvexity and quasiconvexity as we can see in the next result.

Theorem 3.1. Let $X$ be a $\partial$-reliable space and let $f: X \rightarrow \mathbb{R} \cup\{+\infty\}$ be a l.s.c. function. Consider the following assertions

i) $f$ is pseudoconvex.

ii) $f$ is quasiconvex and

$$
(0 \in \partial f(x) \Longrightarrow x \text { is a global minimum of } f) \text {. }
$$

Then, i) implies ii).

And ii) implies i) if $f$ is radially continuous and $\partial f \subset \partial^{\dagger} f$.

Proof. The part ii) $\Rightarrow$ i) is similar to the proof of the corresponding assertion in [1] Theorem 7.1, so we prove only the part i) $\Rightarrow$ ii).

Indeed, by the very definition, it is sufficient to verify that $f$ is quasiconvex. If that was not the case, in view of the lower semicontinuity of $f$, there would exist $x, y \in X, z \in(x, y)$ and $\varepsilon>0$ such that

$$
\text { for all } z^{\prime} \in B_{\varepsilon}(z), \quad f\left(z^{\prime}\right)>\max \{f(x), f(y)\} .
$$


Since $z$ cannot be a local minimum (because apparently $z$ is not a global minimum and for a pseudoconvex function every local minimum is a global), there is some $v$ in $B_{\varepsilon}(z)$ such that $f(v)<f(z)$. Thanks to Theorem 2.1, there exist

$\left(w_{n}\right) \rightarrow \bar{z} \in[v, z)$ and $w_{n}^{*} \in \partial f\left(w_{n}\right)$ such that

$$
\left\langle w_{n}^{*}, z-w_{n}\right\rangle>0 .
$$

But since $z \in(x, y)$, one of the two following cases must holds

$$
\left\langle w_{n}^{*}, x-w_{n}\right\rangle>0 \quad \text { or } \quad\left\langle w_{n}^{*}, y-w_{n}\right\rangle>0 .
$$

Therefore

Hence contradiction follows.

$$
f\left(w_{n}\right) \leq \max \{f(x), f(y)\} .
$$

When $\partial$ is the Clarke-Rockafellar subdifferential $\partial^{C R},[\mathbf{i})$ implies ii)] has been proved by Daniilidis-Hadjisavvas in $[8]$.

In the particular case where $f$ is strictly pseudoconvex we have the following simplified form of Theorem 3.1.

Proposition 3.1. Let $X$ be a $\partial$-reliable space and let $f: X \rightarrow \mathbb{R} \cup\{+\infty\}$ be a l.s.c. function. Consider the following assertions

i) $f$ is strictly pseudoconvex.

ii) $f$ is strictly quasiconvex and

$(0 \in \partial f(x) \Longrightarrow x$ is a global minimum of $f)$.

Then i) implies ii) if $\partial f(x)$ is nonempty for any $x \in X$.

And ii) implies i) if $f$ is radially continuous and $\partial f \subset \partial^{\dagger} f$.

Proof. Let $f$ be a strictly pseudoconvex function, then by Theorem 3.1, the function $f$ is quasiconvex and satisfies the following optimality condition

$$
0 \in \partial f(x) \Longrightarrow(x \text { is a global minimum of } f) .
$$

According to Diewert [9], it suffices to prove that $f$ is radially non-constant. Assume by contradiction that there exists a closed segment $[x, y]$ with $x \neq y$ where $f$ is constant. Let $z \in(x, y)$ and apply the strict pseudoconvexity property to $x$ and $z$, then

$$
(f(z)=f(x)) \Longrightarrow \quad\left(\forall z^{*} \in \partial f(z): \quad\left\langle z^{*}, x-z\right\rangle<0\right) .
$$

Using the same argument for $z$ and $y$ we obtain

$$
(f(z)=f(y)) \Longrightarrow \quad\left(\forall z^{*} \in \partial f(z): \quad\left\langle z^{*}, y-z\right\rangle<0\right) .
$$

Since $\partial f(z)$ is nonempty, it follows that

$$
\text { for all } z^{*} \in \partial f(z), \quad\left\langle z^{*}, x-y\right\rangle<0 \quad \text { and } \quad\left\langle z^{*}, x-y\right\rangle>0 .
$$

A contradiction. Conversely, suppose that $f$ satisfies condition ii) of Proposition 3.1. Then by Theorem 3.1, $f$ is pseudoconvex. Suppose by contradiction that there exist $x \neq y$ in $X$ and $x^{*} \in \partial f(x)$ and $y^{*} \in \partial f(y)$ such that

$$
\left\langle x^{*}, y-x\right\rangle \geq 0 \quad \text { and } \quad f(x) \geq f(y) .
$$


It follows by pseudoconvexity that

$$
\text { for all } z \in[x, y], \quad f(z) \geq f(x) \geq f(y) \text {. }
$$

Since $f$ is quasiconvex, then we have

$$
\text { for all } z \in[x, y], \quad f(z)=f(x) .
$$

So $f$ is not radially non-constant on $X$ (since $f$ is constant on $[x, y]$ ).

Then $f$ is not strictly quasiconvex.

Geometrically we ca see that the subdifferential of a pseudoconvex function $f$ at any point $x \in X$ is a subset of the normal cone $N\left(S_{f}(f(x)), x\right)$ to the sublevel set $S_{f}(f(x))$, more precisely we have :

Proposition 3.2. Let $X$ be a d-reliable space and $f: X \rightarrow \mathbb{R} \cup\{+\infty\}$ be a function that is l.s.c. and pseudoconvex such that $\partial f \subset \partial^{\dagger} f$. Then we have

$$
\text { for all } x \in X, \quad c l\left(\mathbb{R}_{+} \partial f(x)\right) \subset N\left(S_{f}(f(x)), x\right) .
$$

Proof. Suppose for contradiction that there exists $v$ such that

$$
v \in \operatorname{cl}\left(\mathbb{R}_{+} \partial f(x)\right) \text { and } v \notin N\left(S_{f}(f(x)), x\right) .
$$

Without loss of generality we suppose that $v=x^{*} \in \partial f(x)$.

Then, we can find some $y$ in $S_{f}(f(x))$ and $\varepsilon>0$ such that

$$
\text { for all } y^{\prime} \in B_{\varepsilon}(y), \quad\left\langle x^{*}, y^{\prime}-x\right\rangle>0 .
$$

Therefore we have:

$$
\text { for all } y^{\prime} \in B_{\varepsilon}(y), \quad f\left(y^{\prime}\right) \geq f(x) \geq f(y) .
$$

Then $f$ and by the pseudoconvexity of $f$ it is a global minimum of $f$.

On the other hand, since $f^{\dagger}(x, y-x)>0$, there exist $\left(x_{n}\right) \rightarrow x$ and $\left(t_{n}\right) \rightarrow 0_{+}$such that

$$
f\left(x_{n}+t_{n}\left(y-x_{n}\right)\right)>f\left(x_{n}\right) .
$$

By Theorem 3.1, $f$ is quasiconvex and then for $n$ sufficiently large,

$f(y)>f\left(x_{n}\right)$, hence contradiction follows with $y$ is a global minimum of $f$.

Now, recall that an operator $T: X \rightarrow 2^{X^{*}}$ is said to be pseudomonotone if for any $x, y \in X$, the following implication holds:

$$
\left(\exists x^{*} \in T(x):\left\langle x^{*}, y-x\right\rangle>0\right) \Longrightarrow \quad\left(\forall y^{*} \in T(y):\left\langle y^{*}, y-x\right\rangle>0\right) .
$$

The following characterization extends a similar one in $[1,4]$ to larger classes of subdifferentials and functions.

Theorem 3.2. Let $X$ be a $\partial$-reliable space and let $f: X \rightarrow \mathbb{R} \cup\{+\infty\}$ be a l.s.c. and pseudoconvex function with a convex domain such that $\partial f \subset \partial^{\dagger} f$. Consider the following assertions

i) $f$ is pseudoconvex.

ii) $\partial f$ is pseudomonotone.

Then, i) implies ii).

And ii) implies i) if $f$ is radially continuous. 
Proof. The proof of the implication ii) $\Rightarrow \mathbf{i})$ is similar to the proof of the corresponding assertion in $([20]$ Theorem 4.1.)

For the implication $\mathbf{i}) \Rightarrow$ ii), suppose for contradiction that there exist $x, y$ in $X$ and $x^{*} \in \partial f(x), y^{*} \in \partial f(y)$ such that

$$
\left\langle x^{*}, y-x\right\rangle>0 \quad \text { and } \quad\left\langle y^{*}, y-x\right\rangle \leq 0 .
$$

Then, from Proposition $3.2\left\langle x^{*}, y-x\right\rangle>0$ implies that $f(x)<f(y)$, and by the pseudonvexity of $f,\left\langle y^{*}, y-x\right\rangle \leq 0$ implies that $f(y) \leq f(x)$, hence a contradiction.

\section{Maxima of pSeudoconvex functions}

Consider the following maximization problem

$$
(\mathcal{P}) \quad\left\{\begin{array}{cc}
\text { maximize } & f(x), \\
\text { subject to } & x \in C,
\end{array}\right.
$$

where $f$ is supposed to be pseudoconvex, l.s.c. and radially continuous, and $C$ is a nonempty convex set of $X$. For $z \in C$, denote by

Then we have

$$
C_{z}=\{x \in C: \quad f(x)=f(z)\}
$$

Theorem 4.1. Let $X$ be a $\partial$-reliable space and let $f: X \rightarrow \mathbb{R} \cup\{+\infty\}$ be a l.s.c and pseudoconvex function such that for any $x$ in $C, \partial f(x)$ is nonempty and $\partial f(x) \subset \partial^{\dagger} f(x)$. Let $\bar{x} \in C$ be such that

$$
\inf _{C} f<f(\bar{x}) .
$$

Then $\bar{x}$ is a maximum of $f$ on $C$ if and only if

$$
\text { for all } x \in C_{\bar{x}}, \quad \partial f(x) \subset N(C, x) \text {. }
$$

Proof. Suppose that

$$
f(y) \leq f(\bar{x}) \quad \forall y \in C, \quad \text { or } \quad C \subset S_{f}(f(\bar{x}))
$$

By Proposition 3.2 we have:

$$
\text { for all } x \in C_{\bar{x}}, \quad \partial f(x) \subset N\left(S_{f}(f(x)), x\right) \subset N(C, x) \text {. }
$$

Conversely, assume for contradiction that there exists $\bar{z} \in C$ such that

$$
f(\bar{z})>f(\bar{x}) .
$$

By hypothesis, we can find some $z \in C$ with $f(z)<f(\bar{x})$.

From the radial continuity of $f$, there exists some $x_{0} \in(z, \bar{z})$ such that

$$
f\left(x_{0}\right)=f(\bar{x})
$$

Since $z, \bar{z} \in C$ and $\partial f\left(x_{0}\right) \subset N\left(C, x_{0}\right)$ we get

$$
\text { for all } x_{0}^{*} \in \partial f\left(x_{0}\right), \quad\left\langle x_{0}^{*}, z-x_{0}\right\rangle=0 \text {. }
$$

Since $f$ is pseudoconvex then, $f\left(x_{0}\right)=f(\bar{x}) \leq f(z)$, hence contradiction follows with $f(z)<f(\bar{x})$. 
This result is a refinement of Theorem2.1 Hiriart-Urruty and Yu. S. Lediaev [15] where the function is supposed to be convex and continuous.

In the particular case where $\partial$ is the Clarke-Rockafellar subdifferential $\partial^{C R}$ and the set $C$ takes the form

$$
C=\{x \in X: \quad g(x) \leq 0\}
$$

where $g$ is supposed to be pseudoconvex and continuous, and such that there is some $x_{0} \in X$ with $g\left(x_{0}\right)<0$, we have

Proposition 4.1. Let $X$ be a Banach space and $f, g: X \rightarrow \mathbb{R} \cup\{+\infty\}$ be pseudoconvex and continuous with nonempty subdifferentials in $C$, and let $\bar{x} \in C$ be such that

$$
\inf _{C} f<f(\bar{x}) .
$$

Then $\bar{x}$ is a maximum of $f$ on $C$ if and only if, for any $x \in C_{\bar{x}}$

$$
g(x)=0 \quad \text { and } \quad \partial^{C R} f(x) \subset \operatorname{cl}\left(\mathbb{R}_{+} \partial^{C R} g(x)\right) .
$$

Proof. Suppose that $\bar{x}$ is a maximum of $f$ on $C$, let us first show that $\bar{x}$ is on the boundary of $C$. Assume by the contrary that $\bar{x}$ is not on the boundary of $C$. Therefore, there exist $x_{1}, x_{2} \in C$ such that $f\left(x_{1}\right)<f(\bar{x})$ and $\bar{x} \in] x_{1}, x_{2}\left[\right.$. Since $f$ is pseudoconvex and $f\left(x_{1}\right)<f(\bar{x})$ then

$$
\left\langle\bar{x}^{*}, x_{1}-\bar{x}\right\rangle<0, \quad \forall \bar{x}^{*} \in \partial^{C R} f(\bar{x})
$$

From Proposition 3.2 and $f\left(x_{2}\right) \leq f(\bar{x})$, we have

$$
\left\langle\bar{x}^{*}, x_{2}-\bar{x}\right\rangle \leq 0, \quad \forall \bar{x}^{*} \in \partial^{C R} f(\bar{x})
$$

As $\bar{x} \in] x_{1}, x_{2}[$, then (1) and (2) means that

$$
\left\langle\bar{x}^{*}, x_{1}-x_{2}\right\rangle<0 \text { and }\left\langle\bar{x}^{*}, x_{2}-x_{1}\right\rangle \leq 0, \quad \forall \bar{x}^{*} \in \partial^{C R} f(\bar{x})
$$

Since $\partial^{C R} f(\bar{x})$ is nonempty, we get a contradiction. It follows that $\bar{x}$ is on the boundary of $C$, hence $g(\bar{x})=$ $0>g\left(x_{0}\right)$, then by Theorem 4.1 and Proposition 2.2 of [12], we have that

$$
\text { for all } x \in C_{\bar{x}}, \quad \partial^{C R} f(x) \subset N(C, x)=c l\left(\mathbb{R}_{+} \partial^{C R} g(x)\right) \text {. }
$$

Conversely, since for any $x \in C_{\bar{x}}, g(x)=0$, Proposition 2.2 of [12]

$$
N(C, x)=\operatorname{cl}\left(\mathbb{R}_{+} \partial f(x)\right) .
$$

According to Theorem 4.1, $\bar{x}$ is a maximum of $f$ on $C$.

As an illustration of Theorem 4.1, we consider the following function $f: \mathbb{R}^{2} \rightarrow \mathbb{R}$ defined by

$$
f(x, y)=\left\{\begin{array}{cc}
\max \{x, y\} & \text { if } x<0 \text { and } y<0 \\
0 & \text { otherwise }
\end{array}\right.
$$

It is clear that $f$ is continuous and quasiconvex on $\mathbb{R}^{2}$. Let us show that it is pseudoconvex on $\mathbb{R}_{*}^{-} \times \mathbb{R}_{*}^{-}$. Since for any $(x, y) \in \mathbb{R}_{*}^{-} \times \mathbb{R}_{*}^{-}$we have :

$$
\partial f(x, y)=\left\{\begin{array}{cc}
\{(\mu, 1-\mu): \mu \in[0,1]\} & \text { for } x=y \\
\{(0,1)\} & \text { for } x<y \\
\{(1,0)\} & \text { for } x>y
\end{array}\right.
$$


Then $(0,0) \notin \partial f(x, y)$, by Theorem $3.1, f$ is pseudoconvex on the open convex set $\mathbb{R}_{-}^{*} \times \mathbb{R}_{-}^{*}$. Consider the convex set $C=[-2,-1]^{2}$ and $(-1,-1) \in C$, then we can see that

$$
C_{(-1,-1)}=\{(x,-1) ;(-1, y): \quad(x, y) \in C\},
$$

and that for any $(x, y) \in C_{(-1,-1)}$

$$
N(C,(x, y))=\left\{\begin{array}{lc}
\{0\} \times \mathbb{R}^{+} & \text {for }(x, y) \in]-2,-1[\times\{-1\}, \\
\mathbb{R}^{+} \times\{0\} & \text { for }(x, y) \in\{-1\} \times]-2,-1[, \\
\mathbb{R}^{+} \times \mathbb{R}^{-} & \text {for }(x, y)=(-1,-2), \\
\mathbb{R}^{-} \times \mathbb{R}^{+} & \text {for }(x, y)=(-2,-1), \\
\mathbb{R}^{+} \times \mathbb{R}^{+} & \text {for }(x, y)=(-1,-1),
\end{array}\right.
$$

So, $\partial f(x, y) \subset N(C,(x, y))$. By Theorem 4.1, we conclude that $(-1,-1)$ is a global maximum of $f$ on $C$.

\section{Applications to Fractional or quadratic Programming}

First consider the following fractional problem

$$
\left(\mathcal{P}_{1}\right) \quad\left\{\begin{array}{l}
\text { maximize } q(x)=f(x) / g(x), \\
\text { subject to } x \in C .
\end{array}\right.
$$

Where $f$ and $g$ are locally Lipschitz functions on some open convex set $O$ containing the convex set $C$.

Then the function $q$ is locally lipschitz. If we require in addition the following

C1) $f$ is convex and $g$ is concave on $O$,

C2) $f$ is nonnegative and $g$ is positive on $O$,

then $q$ is pseudocopnvex on $O$, indeed, we have :

Proposition 5.1. Let $X$ be a d-reliable Banach space and let $q$ be a function defined as in $\left(\mathcal{P}_{1}\right)$ such that $\partial q \subset \partial^{C R} q$. If $\mathbf{C 1}$ ) and $\mathbf{C 2}$ ) hold, then $q$ is pseudoconvex on $O$.

Proof. For any $\alpha \in \mathbb{R}$, we observe that

$$
S_{q}(\alpha)=S_{h_{\alpha}}(0)
$$

where the function $h$ is defined by

$$
h_{\alpha}(x)=f(x)-\alpha g(x), \quad \forall x \in O .
$$

Since $h_{\alpha}$ is convex, $q$ is quasiconvex.

In order to prove the pseudoconvexity of the function $q$, it suffices to show that if $0 \in \partial q(x)$, then $x$ is necessarily a global minimum of the function $q$.

Consider $x \in O$ such that $0 \in \partial q(x)$, hence

$$
0 \in g(x) \partial^{C R} f(x)-f(x) \partial^{C R} g(x) .
$$

It follows that $0 \in \partial^{C R} h_{q(x)}(x)$, this means that $x$ is a global minimum of $h_{q(x)}$, and since $h_{q(x)}(x)=0$, we have

$$
f(y)-q(x) g(y) \geq 0, \quad \forall y \in O .
$$

Therefore $x$ is a global minimum of $q$ on $O$. 
As an illustration of Theorem 4.1, we study the following linear fractional problem :

$$
\left(\mathcal{P}_{2}\right) \quad\left\{\begin{array}{cc}
\text { maximize } & f(x)=x_{1} /\left(x_{1}+x_{2}\right), \\
\text { subject to } & x=\left(x_{1}, x_{2}\right) \in C=[1,2]^{2} .
\end{array}\right.
$$

By the preceding Proposition, the function $f$ is pseudoconvex on $X_{+}$, where

$$
X_{+}=\left\{x \in \mathbb{R}^{2}: \quad x_{1}+x_{2}>0\right\} .
$$

And since $C$ is a compact subset of the half space $X_{+}$, the maximum of $f$ in $C$ is achieved at some point $\bar{x}=\left(\bar{x}_{1}, \bar{x}_{2}\right)$.

On the other hand, for any $x \in C$, we have

$$
\nabla f(x)=\frac{1}{\left(x_{1}+x_{2}\right)^{2}}\left(\begin{array}{c}
x_{2} \\
-x_{1}
\end{array}\right) .
$$

Then, it is easy to verify that the condition

$$
\nabla f(x) \in N(C, x) \quad \forall x \in C_{\bar{x}},
$$

holds for the point $\bar{x}=(2,1)$, since $C_{\bar{x}}=\{\bar{x}\}$ and $N(C, \bar{x})=\mathbb{R}^{+} \times \mathbb{R}^{-}$. Then by Theorem $4.1, \bar{x}$ is a maximum of $f$ on $C$.

And now consider the quadratic problem

$$
\left(\mathcal{Q}_{1}\right) \quad\left\{\begin{array}{rr}
\text { maximize } & f(x)=\frac{1}{2}\langle A x, x\rangle+\langle a, x\rangle+\alpha, \\
\text { subject to } & x \in C=\left\{x \in \mathbb{R}^{n}: \quad g(x) \leq 0\right\},
\end{array}\right.
$$

where $g$ is a convex, $g(x)=\frac{1}{2}\langle B x, x\rangle+\langle b, x\rangle+\beta$, where $A$ and $B$ are symmetric matrices.

Proposition 5.2. Let $f$ and $g$ be two functions defined as in $\left(\mathcal{Q}_{1}\right)$ such that $g$ is convex and $f$ is pseudoconvex on an open convex containing $C$. Consider $\bar{x} \in C$ such that the following assumptions hold:

i) There is some $x_{0} \in C$ such that $g\left(x_{0}\right)<0$,

ii) There is some $y_{0} \in C$ such that $f\left(y_{0}\right)<f(\bar{x})$,

Then, $\bar{x}$ is a solution of $\left(\mathcal{Q}_{1}\right)$ if and only if for any $x \in C_{\bar{x}}$,

$$
g(x)=0 \quad \text { and } \quad \exists \mu=\mu(x)>0 \quad \text { such that } \quad A x+a=\mu(B x+b) .
$$

The proof is omitted because this Proposition is only another way of stating Proposition 4.1. As an illustration, consider

$$
\left(\mathcal{Q}_{2}\right) \quad\left\{\begin{array}{c}
\text { maximize } \\
\text { subject to } \quad x=\left(x_{1}, x_{2}\right) \in C=\left\{x \in \mathbb{R}^{n}: \quad g(x) \leq 0\right\},
\end{array}\right.
$$

where $g(x)=\frac{9}{4} x_{1}^{2}+\frac{9}{4} x_{2}^{2}-\frac{7}{2} x_{1} x_{2}-2 x_{1}-2 x_{2}+3$.

Then $f$ and $g$ are quadratic functions with associated matrices

$$
A=\left(\begin{array}{cc}
0 & -1 \\
-1 & 0
\end{array}\right) \quad \text { and } \quad B=\left(\begin{array}{cc}
9 / 2 & -7 / 2 \\
-7 / 2 & 9 / 2
\end{array}\right)
$$


Since $B$ is positive semi-definite, $g$ is convex.

On the other hand, $f$ is pseudoconvex on the open convex set

$$
O=\left\{x=\left(x_{1}, x_{2}\right): \quad x_{1}>0, x_{2}>0\right\} .
$$

Indeed, since the sublevel sets of $f$ are convex, $f$ is quasiconvex on the open convex set $O$ with $\nabla f(x) \neq 0$ for any $x \in O$, then by Theorem $3.1, f$ is pseudoconvex on $O$. Moreover, one can also verify that $g(x) \leq 0$ if and only if

$$
\frac{X^{2}}{2}+4 Y^{2} \leq 1
$$

where

$$
X=\frac{\sqrt{2}}{2}\left(x_{1}+x_{2}-4\right) \quad \text { and } \quad Y=\frac{\sqrt{2}}{2}\left(x_{2}-x_{1}\right) .
$$

It follows then that $C$ is included in the the disk of center $(2,2)$ and radius $\sqrt{2}$ and then in $D$. Notice however that $f$ is pseudoconvex and not convex on $C$ as we can check easily with the three points $(1,1)(2,2)$ and $(3,3)$. Consider the point $\bar{x}=\left(\bar{x}_{1}, \bar{x}_{2}\right)=(1,1)$ it satisfies

$$
\left(\begin{array}{cc}
0 & -1 \\
-1 & 0
\end{array}\right)\left(\begin{array}{l}
\bar{x}_{1} \\
\bar{x}_{2}
\end{array}\right)=\bar{\mu}\left(\begin{array}{cc}
9 / 2 & -7 / 2 \\
-7 / 2 & 9 / 2
\end{array}\right)\left(\begin{array}{l}
\bar{x}_{1} \\
\bar{x}_{2}
\end{array}\right)-\left(\begin{array}{c}
2 \bar{\mu} \\
2 \bar{\mu}
\end{array}\right)
$$

with $\bar{\mu}=1>0$; moreover we can check easily that $C_{\bar{x}}=\{\bar{x}\}$. Then $\bar{x}$ is the solution of $\left(\mathcal{Q}_{2}\right)$.

\section{REFERENCES}

[1] D. Aussel, Théorème de la valeur moyenne et convexité généralisée en analyse non régulière, Université Blaise Pascal of Clermont-Ferrand, France, Ph-D Thesis, (1994).

[2] D. Aussel, J. N. Corvellec and M. Lassonde, Mean value property and subdifferential criteria for lower semicontinuous functions. Trans. Amer. Math. Soc. 347, 10, 4147-4161 (1995).

[3] D. Aussel, J. N. Corvellec and M. Lassonde, Subdifferential characterization of quasiconvexity and convexity. J. Convex Anal. $1,2,195-201$ (1994).

[4] D. Aussel, Subdifferential properties of quasiconvex and pseudoconvex functions: unified Approach, J. Optim. Theory Appl. 97, no. 1, 29-45 (1998).

[5] D. Azé, J.-N. Corvellec and R. E. Lucchetti, Variational pairs and applications to stability in nonsmooth analysis, Nonlinear Anal., Theory, Methods and Appl. 49,5, 643-670 (2002).

[6] F. H. Clarke, Optimization and nonsmooth analysis, Wiley-Interscience, New York, (1983).

[7] F. H. Clarke, Yu. S. Ledyaev, R. J. Stern and P. R. Wolenski, Nonsmooth analysis and control theory, Springer-Verlag, New York, (1998).

[8] A. Daniilidis and N. Hadjisavvas, On subdifferentials of quasiconvex and pseudoconvex functions and cyclic monotonicity, Aegean University press, Greece, (1997).

[9] W. E. Diewert, Alternative characterizations of six kinds of quasiconvexity in the nondifferentiable case with applications to nonsmooth programming, Academic Press (1981).

[10] A. Hassouni Sous-différentiel des fonctions quasi-convexes thèse de troisième cycle, Univ P. Sabatier, Toulouse,(1983).

[11] A. Hassouni Quasimonotone multifunctions; applications to optimality conditions in quasiconvex programming Numerical functional analysis and optimization,13:3-43-4, 267-275, Dekker(1992).

[12] A. Hassouni and A. Jaddar, Quasiconvex functions and applications to optimality conditions in nonlinear programming, Appl. Math. Lett.,14, no. 2, 241-244, (2001).

[13] A. Hassouni and A. Jaddar, On generalized monotone multifunctions with applications to optimality conditions in generalized convex programming, J. Inequal. Pure Appl. Math., 4(4), Article 67,(2003).

[14] J.-B. Hiriart-Urruty, Tangent cones, generalized gradients and mathematical programming in Banach spaces, Math. Oper. Res. 4, 79-97 (1979).

[15] J.-B. Hiriart-Urruty and Yu. S. Ledyaev, A note on characterization of the global maxima of a (tangentially) convex function over a convex set, J. Convex Anal., 3, $N^{\circ} 1,55-31$ (1996).

[16] V. L. Levin, Quasi-convex functions and quasi-monotone operators, J. Convex Anal. 2, no. 1-2, 167-172 (1995).

[17] O. L. Mangasarian, Pseudoconvex functions, SIAM J. Control, 3, 281-290 (1965). 
[18] J.-P. Penot, Generalized convex functions in the light of nonsmooth analysis, Lecture notes in Economics and Math. Systems, 429, Springer Verlag, 269-291 (1995).

[19] J.-P. Penot, Are generalized derivatives useful for generalized convex functions? Generalized convexity, generalized monotonicity: recent results (Luminy, 1996), 3-59, Nonconvex Optim. Appl., 27, Kluwer Acad. Publ., Dordrecht, (1998).

[20] J.-P. Penot and P. H. Quang, Generalized convexity of functions and generalized monotonicity of set-valued maps, J. Optim. Th. Appl. 92, 343-356 (1997).

[21] J.-P. Penot and P. H. Sach, Generalized Monotonicity of Subdifferentials and Generalized Convexity, J. Optim. Th. Appl. 94, Issue 1, 251-262 (1997).

[22] R. T. Rockafellar, Generalized directional derivatives and subgradients of nonconvex functions, Canad. J. Math. 32, 257-280 (1980).

[23] S. Schaible and W. T. Ziemba (edi.), Generalized Concavity in Optimization and Economics, Academic Press, New-York, (1981).

[24] A. Strekalovski, On search of global maximum of convex functions on a constraint set, J. of Comp. Mathematics and Math. Physics 33 349-363 (1993).

[25] D. Zagrodny, Approximate mean value Theorem for upper subderivatives, Nonlinear Anal. 12, 1413-1428, (1988). 